\title{
920 MHz ALUMINUM NITRIDE CROSS-SECTIONAL LAMÉ MODE PIEZOELECTRIC MEMS TRANSFORMER WITH HIGH OPEN-CIRCUIT VOLTAGE GAIN IN EXCESS OF 39

\author{
Cristian Cassella, Guofeng Chen, Zhenyun Qian, Gwendolyn Hummel and Matteo Rinaldi
} \\ Northeastern University, Boston, USA
}

\begin{abstract}
In this work we present the first piezoelectric transformer based on a $920 \mathrm{MHz}$ 2-port CLMR. This device, which is dubbed crosssectional Lamé-mode transformer (CLMT), shows a loaded quality factor of $\sim 1000$ and a record high $k_{t}^{2}$ for AIN 2-port resonators in excess of $3.9 \%$, thus leading to $\mathrm{G}_{\mathrm{v}}$ value, in air, close to 39 . Such record high value of $\mathrm{G}_{\mathrm{v}}$, which matches closely the values predicted by both Finite-Element-Methods (FEM) and circuit modelling, is attained through the use of two optimized 3-finger interdigital metal electrodes (IDTs) sandwiching a $4-\mu \mathrm{m}$ thick AlN film. The demonstration of such a high voltage-gain in an AlN piezoelectric transformer enables the development of new strategies to achieve energy awareness and deeper degrees of miniaturization in next generation integrated RF circuits.
\end{abstract}

\section{INTRODUCTION}

The growing demand of service bands in mobile wireless platforms is strengthening the need of adaptive radio-frequency (RF) front-ends. In particular, the use of reconfigurable antennas and filtering banks is key to reduce the size of front-ends without compromising their sensitivity and, consequently, degrading the quality of their baseband operations. However, in order to enable this approach, the development of reconfigurable matching networks is highly desirable to maximize the power-transfer, from filters to antennas and vice versa, for any possible operative scenario.

The use of piezoelectric transformers (PTs) has been proposed as a compact approach to achieve power-matching without the use of lumped low-Q integrated inductors [1],[2].

PTs are 2-port devices enabling maximum power-transfer between two significantly different electrical terminations. The difference between such impedances is set by their voltage-gain $\left(G_{v}\right)$. In addition, integrated PTs reaching high $G_{v}$-values are highly desirable to achieve miniaturized and high conversion efficiency AC-to-DC [3],[4] and DC-to-DC [5],[6],[7] converters .

Aluminum-Nitride (AlN) piezoelectric resonators are promising candidates to replace off-chip Surface-Acoustic-Wave (SAW) devices in commercial wireless platforms. In particular, since such devices can be built through traditional semiconductor processes, they can be monolithically integrated with the rest of the CMOScircuitry. This crucial feature enables fully-integrated RF frontends. Two main AlN-resonator technologies were previously demonstrated: contour-mode resonators (CMRs) [8] and film-bulkacoustic-resonators (FBARs) [9].

AlN CMRs rely on the transduction of longitudinal vibration along the width of an AlN plate through the use of the $e_{31}$ piezoelectric coefficient of AIN. In contrast, AIN FBARs rely on the transduction of longitudinal vibration along the thickness of an AIN plate through the $e_{33}$ piezoelectric coefficient of AlN. As the $e_{33}$ is significantly larger than the $e_{31}$, AlN FBARs show higher electromechanical coupling coefficient $\left(k_{t}^{2}\right)$ than CMRs, thus being preferred for the synthesis of wide-band filters for RF applications. In contrast, as the development of 2-port FBAR-based devices is only possible through a significantly more elaborate fabrication process [7], CMRs are preferred to FBARs when devices with two separate terminations are needed. Consequently, previously reported piezoelectric PTs are mostly based on CMRs. However, as the voltage-gain of PTs is proportional to their $k t^{2}$, PTs based on AIN CMRs can only achieve limited $\mathrm{G}_{\mathrm{v}}$-values.

More recently, AlN cross-sectional Lamé-mode resonators were demonstrated (CLMRs) [10],[11]. CLMRs are piezoelectric resonators formed by two metallic IDTs sandwiching an AIN plate. They rely on the transduction of a Lamé-mode in the cross-section of an AlN plate through the combined use of both the $e_{31}$ and the $e_{33}$ piezoelectric coefficients of AIN. Differently from other existing piezoelectric resonator technologies, CLMRs ate characterized by high- $k_{t}{ }^{2}$ and lithographically determined resonance frequency $\left(f_{\text {res }}\right)$. Therefore, they are suitable for the synthesis of lithographically defined integrated contiguous and not-contiguous pre-select filters for platforms adopting carrier-aggregation (CA).

In this work we report the performance of the first PT-based on the CLMR-technology. This device, which we labeled as crosssectional Lamé-mode transformer (CLMT), operates in the RFfrequency range $(\backsim 920 \mathrm{MHz})$ and shows maximum $G_{v}$-value in excess of 39. Such a record-high value for AlN PTs is enabled by the large input- $k_{t}^{2}(\sim 4 \%)$ achieved by 2-port AIN CLMRs.

\section{CROSS-SECTIONAL LAMÉ-MODE RESONATORS}

CLMTs exploit the unique piezoelectric and electromechanical properties of the recently developed cross-sectional Lamé-mode resonators (CLMRs).

AIN CLMRs use the coherent combination of the $e_{31}$ and $e_{33}$ piezoelectric coefficients of AlN to transduce a Lamé-mode in the cross-section of an AlN plate (Fig. 1). Thanks to the opposite sign of these two piezoelectric coefficients, in-phase charge components are generated by vibration along both the cross-sectional directions (thickness and width) of the plate. As a consequence, the $k_{t}{ }^{2}$ attained by AIN CLMRs is function of both the $e_{31}$ and $e_{33}$ piezoelectric coefficients of AlN and it is higher than the $k_{t}^{2}$ achieved by conventional laterally vibrating AIN resonators such as CMRs.

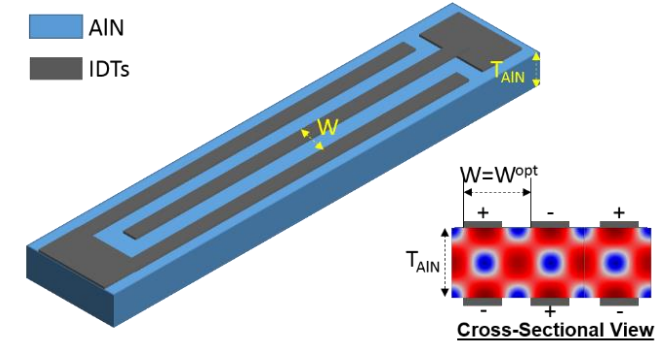

Figure 1: schematic-view of a 3-finger CLMR. The device is formed by two IDTs sandwiching an AlN film. The pitch of the IDTs $(W)$ is selected to be similar to the thickness of the AlN layer $\left(T_{A l N}\right)$. Such choice enables the excitation of high- $k_{t}^{2}$ degenerate or nondegenerate Lamé-modes in plates. The mode-shape relative to the total displacement of the same device, when exciting a nondegenerate Lamé mode, is also reported. 
Similarly to CMRs, CLMRs can be excited through either a Lateral-Field-Excitation (LFE) [12] or a thickness-field-excitation (TFE) approach [6]. LFE CLMRs are formed by one set of IDT patterned on either the top or the bottom surface of an AIN layer. In contrast, TFE CLMRs are formed by two interdigital-metal electrodes sandwiching an AIN film. The IDTs, in both TFE and LFE CLMRs, are needed to produce the excitation electric field in the ross-section of the AlN layer.

As demonstrated in [5], CLMRs achieve maximum $k_{t}{ }^{2}$-value when the pitch of the IDTs (W) is set to a specific value ( $W^{\text {opt }}$ ) similar to thickness $\left(\mathrm{T}_{\mathrm{AIN}}\right)$ of the AlN plate. In fact, in this scenario, a nondegenerate Lamé-mode is excited in the cross-section of the AlN plate. However, due to the capability of exciting high- $k_{t}^{2}$ degenerate cross-sectional Lamé modes [14] in plates, CLMRs can attain high $k_{t}{ }^{2}$ also when $\mathrm{W}$ is slightly different from $\mathrm{W}^{\text {opt }}$. In addition, since $f_{\text {res }}$ depends on $\mathrm{W}$, the transduction of such degenerate modes also enables a significant lithographic tunability of the device operating frequency. This feature is crucial for the implementation of multi-frequency resonators filters and PTs monolithically integrated on the same chip with minimal fabrication complexity.

\section{CROSS-SECTIONAL LAMÉ-MODE PTS}

The high transduction coefficient of AIN CLMRs enables the implementation of 2-port devices with $k_{t}{ }^{2}$ values much higher than the ones of conventional 2-port AlN CMRs [15]. This special feature is key to achieve high performance Cross-Sectional Lamé-Mode transformers (CLMTs). Similarly to CLMRs, CLMTs are formed by two-IDTs sandwiching an AlN-plate. However, differently from CLMRs, only a portion of the IDTs, labeled as input, is connected to the device input-voltage $\left(V_{\text {in }}\right)$. Such portion is responsible for the excitation of the mechanical vibration in the AIN plate, whereas the remaining portion, labeled as output, is adopted to probe the piezoelectrically generated device output-voltage $\left(\mathrm{V}_{\text {out }}\right)$.

A schematic representation of the $920 \mathrm{MHz}$ CLMT of this work is shown in Figure 2.

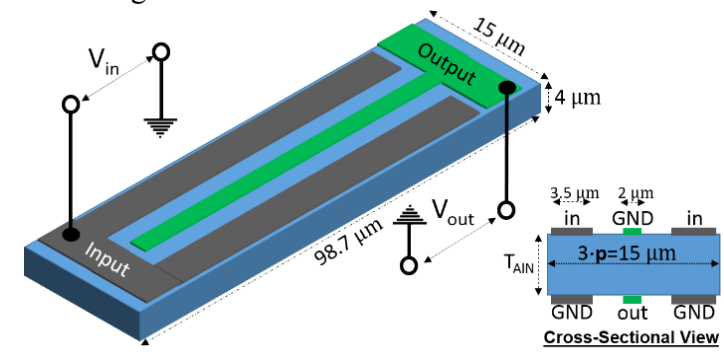

Figure 2: Left) Schematic representations of the CLMT demonstrated in this work. In this device the input-and output-ports use a common ground.

The most important parameter of any piezoelectric transformer is the voltage gain $\left(\mathrm{G}_{\mathrm{v}}\right)$, which is defined as the ratio between the voltage levels at the output and input ports respectively. In particular, to compare the performance of different PT-technologies, the open-circuit voltage-gain $\left(\mathrm{G}_{\mathrm{v}}{ }^{(\mathrm{max})}\right)$ is often used. This parameter expresses the magnitude of the ratio between $V_{\text {out }}$ and $V_{\text {in }}$, when the output port is kept open. The expression of $\mathrm{G}_{\mathrm{v}}{ }^{(\max )}$ is reported in Eq. (1).

$$
G_{v}^{(\max )}=\frac{k_{t}^{2} Q_{\text {load }} C_{i n} \eta \pi^{2}}{8 C_{\text {out }}}
$$

It is important to point out that $\mathrm{G}_{\mathrm{v}}{ }^{(\max )}$ (Eq. (1)) is proportional to the value of the resonator figure-of-merit $\left(\mathrm{FoM} \propto Q \cdot k_{t}^{2}\right)$, which further highlights the advantage of CLMTs over PTs based on any other laterally vibrating piezoelectric resonator technology.

\section{Fabrication Process}

The CLMT of this work is formed by a $4 \mu \mathrm{m}$ thick AIN layer and two $0.1 \mu \mathrm{m}$ thick platinum IDTs placed on the top and bottom surfaces of the AlN film. The choice of using platinum for the bottom IDT was dictated by the need of growing a high quality AIN film. Platinum was also used for the top IDT in order to preserve high acoustic symmetry in the device cross-section.

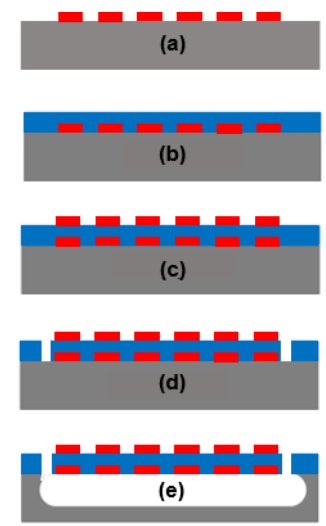

Figure 3: Microfabrication process of the CLMT fabricated in this work: (a) Pt film was deposited on top of Silicon, through a $10 \mathrm{~nm}$ thick Ti layer used as adhesion layer, and patterned through lift-off process; (b) AlN film was deposited on top of the Pt film and vias in the AlN were formed; (c) Pt film was deposited on top of Si and patterned through lift-off process; (d) AlN film was etched through the use of a $\mathrm{SiO}_{2}$ hard mask that was preferred to traditional photoresist mask to attain steeper AlN sidewalls; (e) Si substrate was released by $\mathrm{XeF}_{2}$ isotropic etching.

The devices were fabricated using a four-mask micro-fabrication process (Figure 3): 10/100 $\mathrm{nm}$ of Ti/Pt was deposited and patterned on top of a high resistivity silicon wafer to form the bottom IDT. Next, a $4 \mu \mathrm{m}$ thick AIN film was sputter-deposited.

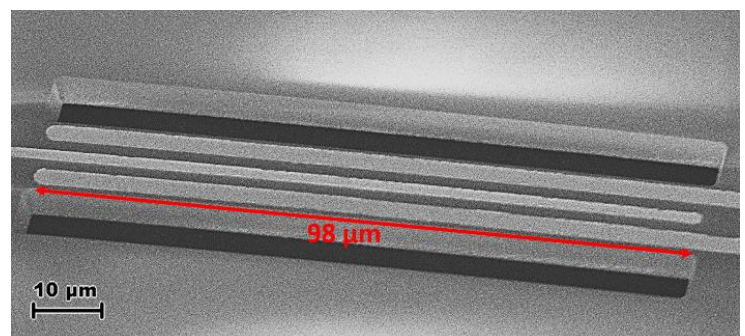

Figure 4: Scanned-Electron-Microscope picture of a fabricated CLMT.

Then, we etched AlN through wet etching to form the vias. Next, 10/100 nm of Ti/Pt was deposited and patterned to form the top IDT. Then, the AlN film was etched by ICP in $\mathrm{Cl}_{2}$ based chemistry to define the width of the AlN plate.

This was done through the use of a hard mask made out of $2 \mu \mathrm{m}$ of $\mathrm{SiO}_{2}$ so as to attain steep AIN sidewall angles $\left(>75^{\circ}\right)$. Finally, the Silicon substrate underneath the resonator was released through $\mathrm{XeF}_{2}$ isotropic etching. A SEM picture of a fabricated CLMT is shows in Figure 4. 


\section{Simulated Performance of 920 MHz 3-fingers CLMTs}

The design of the CLMT proposed in this work was aided by 2DFEA. In particular, we investigated the dependence of $\mathrm{G}_{\mathrm{v}}{ }^{(\mathrm{max})}$ from the pitch of the IDT (i.e. W). The maximum simulated $\mathrm{G}_{\mathrm{v}}{ }^{(\max )}$-values were attained when $\mathrm{W}$ was set to be around $\sim 5 \mu \mathrm{m}$. Such $\mathrm{W}$-value $\left(\mathrm{W}^{(\mathrm{tx})}\right)$ permits to maximize the input- $k_{t}^{2}$ when the device output port is left floating. In addition, we detected a small sensitivity of $\mathrm{G}_{\mathrm{v}}{ }^{(\max )}$ to the width of the metal-strip ( $\mathrm{W}_{\mathrm{el}}{ }^{\text {(out) }}$ ) forming the output portion of the IDTs. So, in the attempt of attaining the highest $\mathrm{G}_{\mathrm{v}}{ }^{(\max )}$-value, $\mathrm{W}_{\mathrm{el}}{ }^{\text {out })}$ was chosen to be almost half $\left(\mathrm{W}_{\mathrm{el}}{ }^{\text {(out) }}=2 \mu \mathrm{m}\right)$ of the width of the metal strips forming the input portion of the IDTs $\left(\mathrm{W}_{\mathrm{el}}{ }^{(\mathrm{in})}=3.5\right.$ $\mu \mathrm{m})$. As verified by FEA, such choice enables a $\sim 10 \%$ improvement in the $\mathrm{G}_{\mathrm{v}}{ }^{(\max )}$-value with respect to the scenario in which $\mathrm{W}_{\mathrm{el}}{ }^{\text {(out) }}$ were be the same than $\mathrm{W}_{\mathrm{el}}{ }^{(\mathrm{in})}$. Such improvement is originated from a slight increase of the device $k_{t}^{2}$. The 2D-FEA simulated $\mathrm{G}_{\mathrm{v}^{-}}$and input-admittance $\left(\mathrm{Y}_{\text {in }}\right)$ distributions as functions of frequency are reported in Figure 5. As evident, a $k_{t}^{2}$ in excess of $4.1 \%$ was estimated by 2D-FEA. Such a high $k_{t}{ }^{2}$-value enables a $\mathrm{G}_{\mathrm{v}}{ }^{(\mathrm{max})}$-value

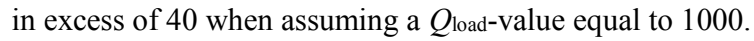

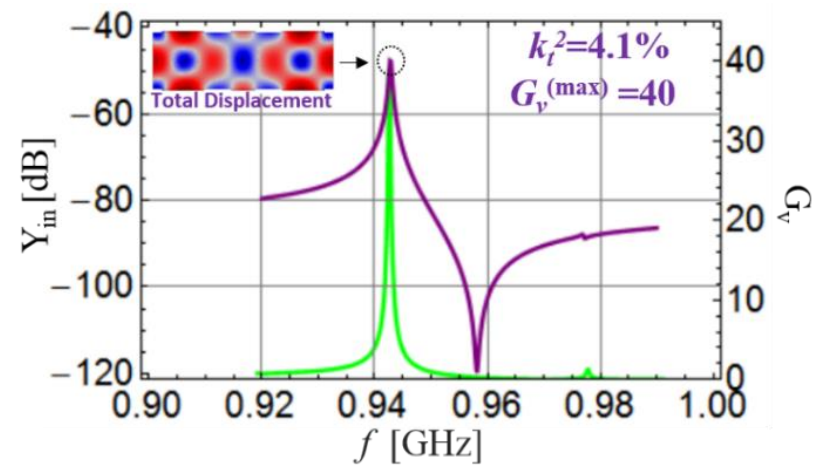

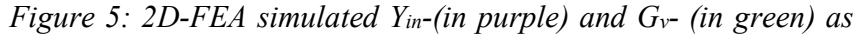
functions of the frequency $(f)$. The simulated responses were computed by assuming a $Q_{\text {load }}$ equal to 1000 . The resonant modeshape of the total displacement is also shown. As expected, the device displaces less underneath the output-port than underneath its input-port. This is due to the transformation mechanism explaining the origin of the voltage amplification in piezoelectric transformers.

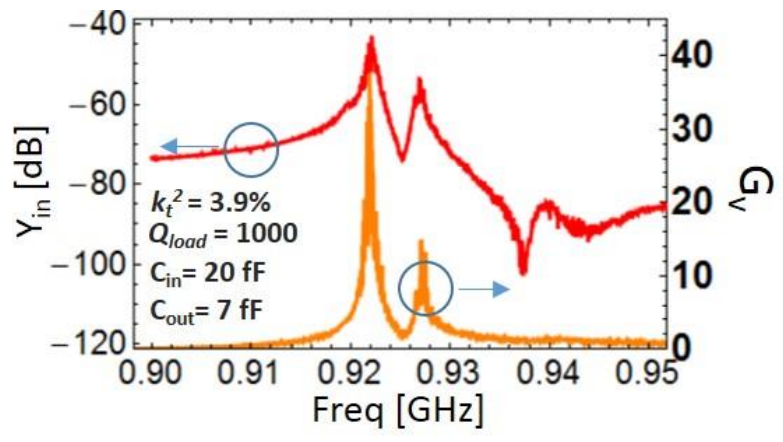

Figure 6: In red) measured $Y_{\text {in }}$ relative of the fabricated CLMT; in black) analytically predicted $Y_{\text {in }}$ using the $Q_{\text {load }}, C_{i n}, C_{o u t}, k_{t}^{2}$ and $\eta$ values measured experimentally. In orange) measured $G_{v}$ of the fabricated CLMT; in blue) analytically predicted $G_{v}(E q .3)$ using the $Q_{\text {load, }} C_{i n}, C_{o u t}, k_{t}{ }^{2}$ and $\eta$ values measured experimentally.

\section{Measured Performance of $920 \mathrm{MHz}$ 3-finger CLMTs}

We experimentally characterized the performance of a fabricated 3-finger $920 \mathrm{MHz}$ TFE CLMT (Figure 4). The device was tested, in air, in a RF probe station using GSG-probes. Its performance are reported in Figure 6. As evident, values of $k_{t}{ }^{2}, Q_{\text {load }}$ and $G_{v}{ }^{(\max )}$ in excess of $4 \%, 1000$ and 39 were directly extracted from the measured Y-matrix. However, a small variation of $f_{\text {res }}$ was measured with respect to its predicted value through FEA. This discrepancy is due to fabrication uncertainties associated with the actual thickness of the IDTs with respect to the value considered in the finite-element simulation (Figure 5).

\section{CONCLUSION}

We report the performance of the first piezoelectric transformer (PT) based on the cross-sectional Lamé-mode resonator technology. The device, which we labeled as a cross-sectional Lamé-mode transformer (CLMT), operates around $920 \mathrm{MHz}$ and shows a recordhigh open-circuit voltage-gain $\left(\mathrm{G}_{\mathrm{v}}{ }^{(\mathrm{max})}\right)$ in excess of 39 . Such a high $\mathrm{G}_{v}{ }^{(\max )}$-value demonstrates the ability of CLMTs to form low-loss narrow-band matching network and voltage-amplification stages in next generation miniaturized AC-to-DC and DC-to-DC converters.

\section{ACKNOWLEDGMENT}

The authors wish to thank the staff of the George J. Kostas Nanoscale Technology and Manufacturing Research Center at Northeastern University for assistance with device fabrication. This work was supported by DARPA MTO under the RF-FPGA (N66001-14-1-4011) and N-ZERO (HR0011-15-C-0138) Programs. The authors wish to thank Dr. Roy Olsson for the support.

\section{REFERENCES}

[1] A. M. Flynn and S. R. Sanders, "Fundamental limits on energy transfer and circuit considerations for piezoelectric transformers," IEEE Trans. Power Electron., vol. 17, no. 1, pp. 8-14, Jan. 2002.

[2] C. Y. Lin and F. C. Lee, "Design of a piezoelectric transformer converter and its matching networks," in , 25th Annual IEEE Power Electronics Specialists Conference, PESC '94 Record, 1994, pp. 607-612 vol.1.

[3] O. Garcia, J. A. Cobos, P. Alou, R. Prieto, and J. Uceda, “A simple single-switch single-stage AC/DC converter with fast output voltage regulation," IEEE Trans. Power Electron., vol. 17, no. 2, pp. 163-171, Mar. 2002.

[4] J. Sebastian, A. Fernandez, P. J. Villegas, M. M. Hernando, and M. J. Prieto, "New active input current shapers to allow AC-to-DC converters with asymmetrically driven transformers to comply with the IEC-1000-3-2," IEEE Trans. Power Electron., vol. 17, no. 4, pp. 493-501, Jul. 2002.

[5] M. Nymand and M. A. E. Andersen, "High-Efficiency Isolated Boost DC \#x2013;DC Converter for High-Power Low-Voltage Fuel-Cell Applications," IEEE Trans. Ind. Electron., vol. 57, no. 2, pp. 505-514, Feb. 2010.

[6] C. S. Moo, W. M. Chen, and H. K. Hsieh, "An electronic ballast with piezoelectric transformer for cold cathode fluorescent lamps," in IEEE International Symposium on Industrial Electronics, 2001. Proceedings. ISIE 2001, 2001, vol. 1, pp. 36-41 vol.1.

[7] O. Ohnishi, H. Kishie, A. Iwamoto, Y. Sasaki, T. Zaitsu, and $\mathrm{T}$. Inoue, "Piezoelectric ceramic transformer operating in thickness extensional vibration mode for power supply," in Ultrasonics Symposium, 1992. Proceedings., IEEE 1992, 1992, pp. 483-488 vol.1.

[8] G. Piazza, "Piezoelectric Aluminum Nitride Vibrating Contour-Mode MEMS Resonators," Berkeley, California, 2005. 
[9] R. C. Ruby, P. Bradley, Y. Oshmyansky, A. Chien, and I. Larson J. D., "Thin film bulk wave acoustic resonators (FBAR) for wireless applications," in 2001 IEEE Ultrasonics Symposium, 2001, vol. 1, pp. 813-821 vol.1.

[10] C. Cassella, G. Chen, Z. Qian, G. Hummel, and M. Rinaldi, "Aluminum Nitride Cross-Sectional Lame Mode Resonators," J. Microelectromechanical Syst., Accepted for publication 2015.

[11] C. Cassella, Z. Qian, G. Hummel, and M. Rinaldi, " $1.02 \mathrm{GHz}$ cross-sectional Lam?? mode resonator with high KT2 exceeding 4.6\%," IEEE 29th Int. Conf. Micro Electro Mech. Syst. MEMS, p. Pages-659, 2016.

[12] Y. Hui, Z. Qian, and M. Rinaldi, “A $2.8 \mathrm{GHz}$ combined mode of vibration aluminum nitride MEMS resonator with high figure of merit exceeding 45," in European Frequency and Time Forum International Frequency Control Symposium (EFTF/IFC), 2013 Joint, 2013, pp. 930-932.

[13] C. Cassella, Z. Qian, G. Hummel, and M. Rinaldi, "1.02 GHz cross-sectional Lam?? mode resonator with high KT2 exceeding 4.6\%," IEEE 29th Int. Conf. Micro Electro Mech. Syst. MEMS, p. Pages-659, 2016.

[14] M. Al-Helaly, "The vibrational spectra of rectangular plates," Ph.D., University of Aston, Birmingham, 1981.

[15] M. Rinaldi, C. Zuniga, C. Zuo, and G. Piazza, "Super-highfrequency two-port AlN contour-mode resonators for RF applications," IEEE Trans. Ultrason. Ferroelectr. Freq. Control, vol. 57, no. 1, pp. 38-45, Jan. 2010.

\section{CONTACTS}

Cristian Cassella, c.cassella@neu.edu, Matteo Rinaldi, rinaldi@ece.neu.edu 